\title{
Glycine oligomerization up to triglycine by shock experiments simulating comet impacts
}

\author{
HARUNA SUgAHARA and KOICHI MIMURA* \\ Department of Earth and Environmental Sciences, Graduate School of Environmental Studies, Nagoya University, \\ Nagoya 464-8601, Japan
}

(Received August 2, 2013; Accepted October 15, 2013)

\begin{abstract}
We conducted shock experiments simulating comet impacts to assess the feasibility of peptide synthesis by such a process. We used frozen mixture of the amino acid glycine, water ice, and silicate (forsterite) as the starting material and applied impact shocks ranging from 4.8 to $26.3 \mathrm{GPa}$ using a vertical propellant gun under cryogenic conditions (77 K). The results show that amino acid oligomerization up to trimers can be achieved. Further, linear peptides (dipeptide and tripeptide forms), which are important materials for the further elongation of peptide chains, were obtained in yields of one or two magnitudes greater than that of cyclic peptide form (diketopiperazine). These results contrast with those by Blank et al. (2001) for shock experiments of amino acid solutions at room temperature, which showed the synthesis of a comparable amount of diketopiperazines to that of the linear peptides. Thus, the existence of cryogenic conditions at the point of impact shock may be critical for the formation of linear peptides. Our results demonstrate that comet impacts could have supplied a significant amount of linear peptides on the early Earth and other extraterrestrial bodies.
\end{abstract}

Keywords: comet impacts, amino acids, peptides, shock experiments, origins of life

\section{INTRODUCTION}

Abiotic peptide synthesis might have played a key role in the chemical evolution that led to the emergence of life. Not only are peptides the building blocks of life, but they also most likely played important roles as catalysts in the formation of biomolecules on the primitive Earth. Polypeptides are known to catalyze the oligomerization of nucleotides, which can lead to the development of genetic material (Barbier et al., 1993); additionally, polypeptides help certain RNA-related molecules to function efficiently (Shimizu, 1995; Kochavi et al., 1997; van der Gulik et al., 2009). Moreover, some chiral dipeptides catalyze the stereoselective synthesis of biomolecules (Weber and Pizzarello, 2006; Pizzarello and Weber, 2010) and may be responsible for the homochirality of biomolecules that is a distinctive feature of terrestrial life.

Several reaction mechanisms of abiotic peptide synthesis in various early terrestrial environments have been proposed, including thermal synthesis at submarine hydrothermal vents (e.g., Imai et al., 1999; Corliss et al., 1981; Shock, 1992; Huber et al., 2003; Martin et al., 2008; Lemke et al., 2009) and volcanic sites (e.g., Fox, 1956; Fox and Harada, 1958; Harada and Fox, 1958), synthesis

\footnotetext{
*Corresponding author (e-mail: mimura@eps.nagoya-u.ac.jp)

Copyright @ 2014 by The Geochemical Society of Japan.
}

by irradiation with ultraviolet light (Simakov et al., 1996; Tanaka et al., 2008), high-energy protons (6.6 MeV) (Simakov et al., 1997), and $30 \mathrm{keV}$ ions of $\mathrm{Ar}^{+}$and $\mathrm{N}^{+}$ (Wang et al., 2007), synthesis using activated amino acid derivatives (e.g., Leuchs, 1906; Oró and Guidry, 1960; Yanagawa et al., 1984; Deming, 2006; Schreiner et al., 2009), and synthesis utilizing the catalytic ability of mineral surfaces to adsorb amino acids (e.g., Bernal, 1951; Flores and Leckie, 1973; Bujdák et al., 1995; Huber and Wächtershäuser, 1998; Lambert, 2008).

On the early Earth, shock synthesis by the impacts of extraterrestrial bodies also may have been significant. The impact frequency of extraterrestrial bodies on the early Earth was significantly higher than at present day (e.g., Chyba and Sagan, 1992; Ryder, 2002; Morbidelli et al., 2012). Impact shocks can cause reactions that transform simple organic materials into more complex ones (Sugisaki et al., 1994; Blank et al., 2001; Mimura and Toyama, 2005; Furukawa et al., 2009; Goldman et al., 2010). It may be possible that amino acids present in extraterrestrial bodies could oligomerize into peptides when impacted upon the early Earth. In fact, more than 80 different amino acids were found in carbonaceous chondrites (e.g., Kvenvolden et al., 1970; Oró et al., 1971; Cronin and Pizzarello, 1983; Ehrenfreund et al., 2001; Botta et al., 2007), with their highest total concentrations reaching $250 \mathrm{ppm}$ (Martins et al., 2007). Although cometary amino acids are not well known, they are also expected 


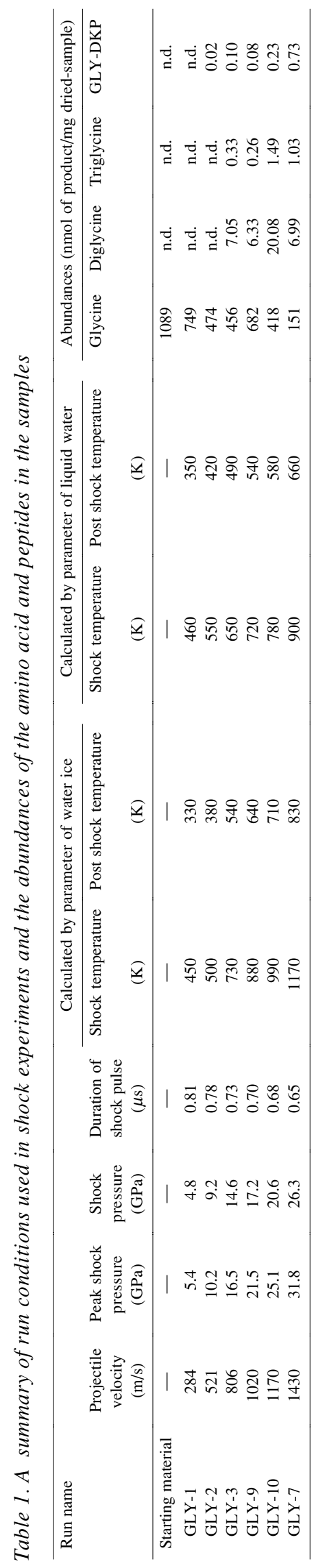

to be present with abundant concentrations (Ehrenfreund and Charnley, 2000; Charnley et al., 2002). This fact is also suggested by laboratory experiments carried out on simulations of interstellar ice analogs, which resulted in the production of various amino acids; accordingly, their relationships to cometary amino acids were proposed (e.g., McPherson et al., 1987; Bernstein et al., 2002; Muñoz Caro et al., 2002; Kobayashi et al., 2004; Holton et al., 2005). The detection of glycine in cometary grains confirmed the existence of an amino acid in comets (Elsila $e t$ al., 2009). Blank et al. (2001) conducted shock experiments on aqueous solutions of amino acids at room temperature to examine the possibility of peptide synthesis by comet impacts. Although the authors identified dipeptides and diketopiperazines as products, their work was preliminary, and data regarding the amount of shocksynthesized peptides was not reported. Further, their experimental results cannot be directly compared with natural comet impacts because the authors did not use water ice but liquid water. Because comets are frozen mixtures of water ice and silicates, such experiments should be conducted under the same conditions.

In the present study, we conducted shock experiments simulating comet impacts to examine the possibility of peptide synthesis by comet impacts as an alternative method to supply peptides to the early Earth. To reproduce successfully the conditions present in comet impacts, we selected solid frozen mixtures of an amino acid (glycine), water ice, and silicate as the starting material and imparted impact shocks at cryogenic conditions. We are the first to report that amino acid oligomerization up to the trimer form occurs as a result of impact shock experiments. We also conducted a quantitative analysis of the shock-synthesized peptides and determined the yields of three types of peptides (diglycine, triglycine, and diketopiperazine). Our results demonstrate that glycine oligomerization, up to at least the trimer form, can occur in natural comet impacts.

\section{Materials ANd Methods}

\section{Starting materials}

We prepared a mixture of glycine (Kishida Chemical, $>99.0 \%$ purity), water ice, and forsterite (Marusu Glaze Industry, $<1.5 \mu \mathrm{m}$ in diameter) to imitate cometary material, used as the starting material. Glycine was detected in the cometary grains returned by NASA's Stardust spacecraft (Elsila et al., 2009), and forsterite is considered to be one of the major silicates in comets (e.g., Tomeoka $e t$ $a l ., 2008)$. We prepared a simple mixture of these components for our experiments to examine the chemical species of the peptides formed by impact shocks and their yields. The mixing ratio was glycine/water ice/olivine $=$ $0.1 / 0.8 / 1.0$, which is based on the composition of inter- 

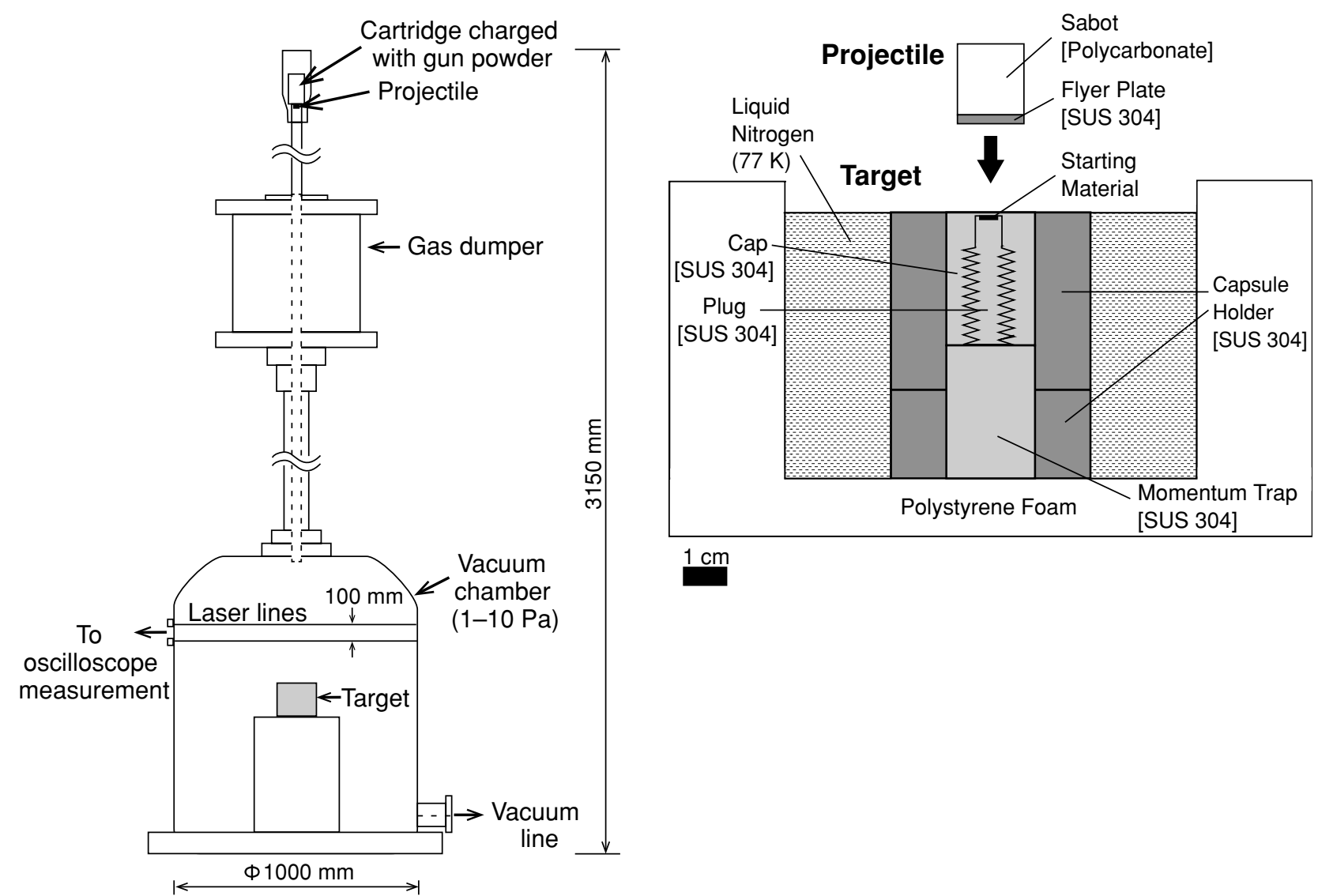

Fig. 1. Vertical propellant gun (A) and a schematic illustration of a target assembly for shock experiment at cryogenic conditions (77 K) (B).

stellar dust (organics/water ice/silicates $=1.4 / 1.3 / 1.0$ ) (Greenberg and Li, 1999). The abundance of glycine was assumed to be approximately $10 \%$ of the organic fraction in interstellar dusts because the abundance in comets is unknown. The precise concentration of glycine used in the starting material is shown in Table 1 . The starting material was prepared by dissolving glycine into liquid water and mixing with forsterite powder at room temperature. Then, the mixture was filled into a sample capsule and was put in a freezer until the shock experiments were performed. The porosity of the starting material was considered to be zero because the fraction of water was significantly larger than that of forsterite, and the starting material was frozen after it was sealed into the capsule.

\section{Shock experiments at cryogenic conditions}

Shock experiments were conducted based on the procedure established in our previous studies (Mimura et al.,
2003). For the experiments, a vertical propellant gun was used to launch a projectile into a target assembly that was placed in the gun's vacuum chamber (1-10 Pa) (Fig. 1A). To calculate the shock pressure and temperature, the velocity of the projectile just before reaching the target assembly was measured optically using lasers. Two laser lines were aligned in parallel in the chamber near the surface of the target assembly, and the crossing time between two laser lines by the projectile was measured using an oscilloscope, from which the velocity of the projectile was calculated. The target assembly consists of three components, including a capsule, a capsule holder, and a momentum trap (Fig. 1-B). The capsule is divided into two parts, a plug and a cap. A small pit (4 $\mathrm{mm}$ in diameter and $0.8 \mathrm{~mm}$ in depth) is located on the top of the plug, and the starting material is placed in this pit. These components are all made of stainless steel (SUS 304). The target assembly was placed in a container of polystyrene foam filled with liquid nitrogen and was set into the gun 
Table 2. Hugoniot Equation of State parameters $\left(U_{s}=C_{0}+S \Delta U_{p}\right)$ used to calculate shock pressure and temperature

\begin{tabular}{lccll}
\hline Material & $\rho_{0}\left[\mathrm{~g} / \mathrm{cm}^{3}\right]$ & $C_{0}[\mathrm{~m} / \mathrm{s}]$ & $S$ & Reference \\
\hline Water ice (ice Ih) & 0.93 & 3000 & 1 & Stewart and Ahrens (2005) \\
Liquid water & 0.998 & 1700 & 1.44 & Stewart and Ahrens (2005) \\
SUS 304 & 7.89 & 4580 & 1.49 & Marsh (1980) \\
\hline
\end{tabular}

chamber to conduct the shock experiments at cryogenic conditions $(77 \mathrm{~K})$.

\section{Chemical analysis}

Shocked samples were recovered from capsules and analyzed using the following procedures, based on Shimoyama and Ogasawara (2002). The shocked samples were divided into two portions. One portion was used for an analysis of the remaining glycine, and the other was used for an analysis of the synthesized peptides. The portion for peptide analysis was further divided into two fractions, one for linear peptide analysis, and the other for cyclic peptides after extraction. Glycine was extracted with Milli-Q water (UV-sterilized Milli-Q water). The extracted sample solution for glycine analysis was dried and derivatized by $1.25 \mathrm{M} \mathrm{HCl}$-isopropanol (Fluka Chemical), and then trifluoroacetic anhydride (TFAA) (Tokyo chemical industry) to make acylated TFA-GlyOiPr ester. The peptides were extracted using methanol and Milli-Q water from the shocked samples and were separated into a linear peptide (dipeptide and tripeptide) fraction and a cyclic peptide (diketopiperazine) fraction using a cation exchange column (Dowex 50W-X8 $\left(\mathrm{H}^{+}\right)$). After the sample solutions were dried, the linear peptide fraction was derivatized to acylated TFA-Gly-OiPr ester. For the derivatization of cyclic peptide fraction, N-tertButyldimethylsilyl-N-methyltrifluoroacetamide with $1 \%$ tert-Butyldimetylchlorosilane (Sigma-Aldrich) that was dissolved into distilled acetonitrile was prepared to yield tert-butyldimethylsilyl derivatives. The samples derivatized by each method were analyzed by a gas chromatograph-mass spectrometry (GC-MS) for the identification of peptides and by a gas chromatograph-flame ionization detector (GC-FID) for quantitative analysis.

\section{RESULTS}

Calculation of the parameters related to impact shock condition

Several parameters related to the shock experiments, such as shock pulse duration, shock pressure, shock temperature, and post shock temperature, were calculated based on the property of the materials used and the projectile velocity. The projectile velocities measured in the experiments ranged from $284 \mathrm{~m} / \mathrm{s}$ to $1430 \mathrm{~m} / \mathrm{s}$. The
Hugoniot data used for the calculations are summarized in Table 2.

Shock pulse duration is determined by the travel time of a shock wave from the impact surface of the flyer plate to the free surface of the plate, then reflected back to the impact surface as a rarefaction wave. Thus, the shock pulse duration $\left(t_{p}\right)$ can be calculated with the following equation (Larouche et al., 1981; Orava and Wittman, 1975):

$$
t_{p}=d^{f}\left(\frac{1}{U_{s}^{f}}+\frac{\rho_{0}^{f}}{\rho^{f} C^{f}}\right)
$$

where $d^{f}$ is the thickness of the flyer plate, $U_{s}^{f}$ is the velocity of the shock wave, $\rho_{0}^{f}$ is the density at $0 \mathrm{GPa}, \rho^{f}$ is the density at a particular pressure, and $C^{f}$ is the sound velocity at a particular pressure. The superscript $f$ denotes that the parameter is related to the flyer plate. The value of $C^{f}$ (SUS 304) at each pressure was taken from Duffy and Ahrens (1997). The calculated shock pulse durations were $0.65-0.81 \mu$ s (Table 1 ).

The shock pressures of the samples were calculated using an impedance matching method (Meyers, 1994) based on the measured projectile velocity, the Hugoniot data of the starting material, the flyer plate (SUS 304), and the capsule (SUS 304) (Marsh, 1980) and under consideration of multiple shock compression. Because the starting material was a mixture of water ice and forsterite, the Hugoniot of the starting material was derived from a mass average of the water ice (or liquid water) and forsterite Hugoniots (Meyers, 1994). In the experiment in which multiple shock compression was applied, the shock wave generated by projectile impact propagates through the upper wall of the cap (SUS 304) and moves into the sample. Because the shock impedance of the sample is lower than that of the surrounding capsule (SUS 304), shock wave reverberation occurs in the sample, which is sandwiched between the upper wall of the cap (SUS 304) and the bottom of the plug (SUS 304). The reverberation generates the shock condition. The reverberated frequencies generated during the shock pulse duration were three to four times in the experiments. The shock pressures achieved in the samples were 4.8-26.3 GPa (Table 1), which were $80-90 \%$ of the peak shock 
pressures, i.e., the maximum pressures achievable by the impact of a projectile (SUS 304) on the target (SUS 304).

The shock temperature was calculated by the following equation (Meyers, 1994):

$$
\begin{aligned}
& T=T_{0} \exp \left[\left(\frac{\gamma_{0}}{V_{0}}\right)\left(V_{0}-V\right)\right]+\frac{\left(V_{0}-V\right)}{2 C_{v}} P \\
& +\frac{\exp \left[\left(-\gamma_{0} / V_{0}\right) V\right]}{2 C_{v}} \int_{V_{0}}^{V} P \cdot \exp \left(\frac{\gamma_{0}}{V_{0}}\right) V\left[2-\left(\frac{\gamma_{0}}{V_{0}}\right)\left(V_{0}-V\right)\right] d V
\end{aligned}
$$

where $P, T, V$ are the shock pressure, the shock temperature, and the specific volume, respectively. The subscript 0 denotes the value at the initial condition. $\gamma$ is the MieGrueneisen parameter, and $C_{v}$ is the heat capacity at constant volume. The shock temperature was calculated based on multiple shock compression. The increase in temperature caused by each reverberation of the shock wave was added step-wise. It should be noted that the shock temperature in multiple shock compression is lower than that of single shock compression because the increase in internal energy is smaller under multiple shock compression than single shock compression (e.g., Mashimo et al., 1980). Although the starting material was a mixture of water ice and forsterite, nonetheless it can be treated as pure water ice for the shock temperature calculation, according to Kraus et al. (2010). They revealed that the shock temperature of a mixture of water ice and quartz is in good agreement with the Hugoniot temperature of pure water ice. The specific volume at each shock pressure was taken from the Hugoniot data generated by Stewart and Ahrens (2005) and the references provided therein. Because the Hugoniot of ice enters the liquid water fields at 9.2 GPa (Stewart and Ahrens, 2005), we applied the parameter of liquid water from $9.2 \mathrm{GPa}$. The values of $C_{v}$ are $1390 \mathrm{~J} \mathrm{~kg}^{-1} \mathrm{~K}^{-1}$ for ice (Chizhov, 1993) and $3420 \mathrm{~J}$ $\mathrm{kg}^{-1} \mathrm{~K}^{-1}$ for liquid water (Stewart and Ahrens, 2005). The $\gamma$ values of ice and liquid water were referred to by Leadbetter (1965) and Stewart and Ahrens (2005), respectively. Hence, in this work, the shock temperatures were calculated to be 450-1170 K (Table 1). These calculated temperatures are most likely higher than the real shock temperature because we have not considered the latent heat related to the phase change from ice to liquid. Stewart and Ahrens (2005) reported that the difference between the Hugoniot temperature of liquid water and water ice is not significant and becomes smaller in the high-pressure region ( $>10 \mathrm{GPa}$ ) of the ice Hugoniot. Then, if we recalculate the shock temperatures assuming that the starting material was made of liquid water (298 K) alone, the Hugoniot temperatures are calculated as 460-900 K.
These temperatures are a realistic representation of the shock temperatures of our experiments. Based on this calculation, the shock temperature of our experiments at 20.6 GPa was estimated to be $990 \mathrm{~K}$. This value is comparable to the value of $870 \mathrm{~K}$ at $21.0 \mathrm{GPa}$ generated in Blank et al. (2001), in which the authors performed shock experiments of liquid water.

We also calculated the post shock temperature, which is determined as the temperature after the pressure has returned zero from the shock state. The calculation was performed based on the following equation, assuming isentropic release (Meyers, 1994)

$$
T_{2}=T_{1} \exp \left(-\int_{V_{1}}^{V_{2}} \frac{\gamma}{V} d V\right)
$$

In the equation, $T_{2}$ is the post shock temperature, and $T_{1}$ is the shock temperature. $V_{2}$ is the specific volume after release, which is approximated as the specific volume of the initial state, and $V_{1}$ is the volume in the shock state. The calculated post shock temperatures in the experiments were $330-830 \mathrm{~K}$. If we recalculate the post shock temperatures based on the shock temperature using the parameters of liquid water, values of 350-660 K are obtained (Table 1). Considering the process of thermal diffusion, it takes several seconds for the sample to return to ambient temperature from the post shock temperature.

\section{Results of the chemical analysis}

Diglycine and triglycine were detected in the shocked samples with shock pressures above $14.6 \mathrm{GPa}$, and glycine-diketopiperazine was determined in those above 9.2 GPa. The peptides were identified by comparison of their retention times and mass fragmental patterns with those of purchased standard materials (Fig. 2). The yields of peptides (mol percent of product/starting glycine) and the survival ratio of glycine against shock pressure are shown in Fig. 3. No peptide was detected in shocked samples with shock pressures below 9.2 GPa, demonstrating that the detected peptides were produced by impact shock and not contamination during the experimental process. The yields of diglycine and triglycine show initial increases of up to $1.8 \mathrm{~mol} \%$ and $0.14 \mathrm{~mol} \%$, respectively, at 20.6 GPa (Figs. 3-A and B) with the increase of shock pressure. Then, the yields declined to $0.64 \mathrm{~mol} \%$ and 0.09 mol\%, respectively, at $26.3 \mathrm{GPa}$. The yield of glycinediketopiperazine increases to $0.07 \mathrm{~mol} \%$ at $26.3 \mathrm{GPa}$ with the increase of shock pressure (Fig. 3-C). The survival ratio of glycine, in contrast, decreases with shock pressure to $13.8 \mathrm{~mol} \%$ at $26.3 \mathrm{GPa}$ (Fig. 3-D). Compared to the peptide yields, the decreasing ratio of glycine was larger than the yields. Thus, not all of the glycine was changed into peptides. Most of the initial glycine most likely decomposed during the impact shock, transform- 

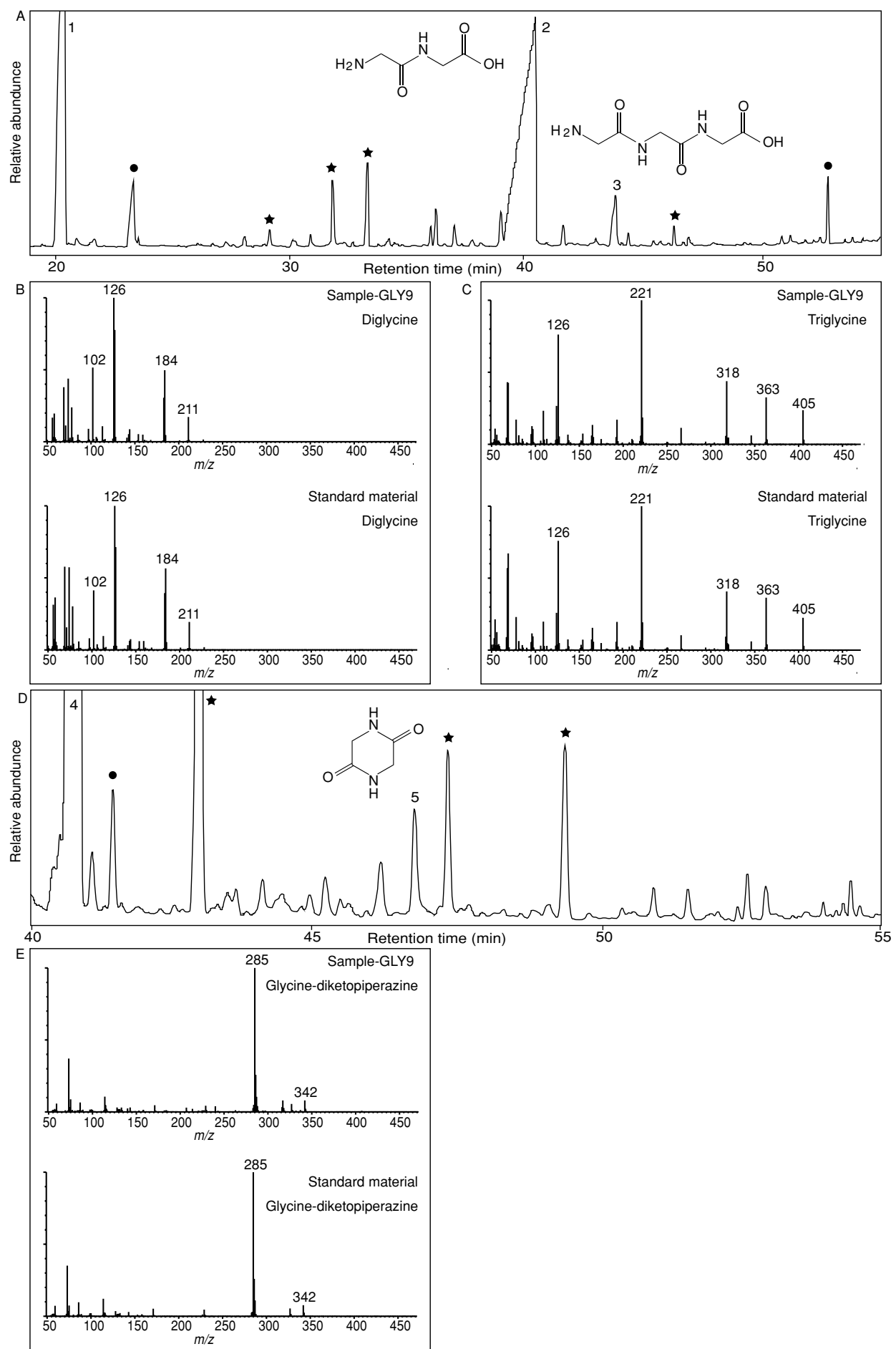

Fig. 2. Total ion chromatograms of the sample (GLY-9) shocked at 17.2 GPa, as a representative spectrum of the shocked samples and the mass fragmental patterns of each peptide peak, compared with those of the purchased standard materials. Chromatograms of the linear peptide fraction $(A)$ and the cyclic peptide fraction $(D)$ are shown. The numbered peaks denote (1) the internal standard (norvaline), (2) diglycine, (3) triglycine, (4) the internal standard (norvaline), and (5) glycinediketopiperazine. The solid stars are background materials that accompany the derivatization process, and the solid circles are materials obtained during other procedures, such as extraction. The mass fragmental patterns of diglycine $(B)$, triglycine $(C)$, and glycine-diketopiperazine (E) of shocked sample (GLY-9) with the corresponding purchased standard materials are also shown. 

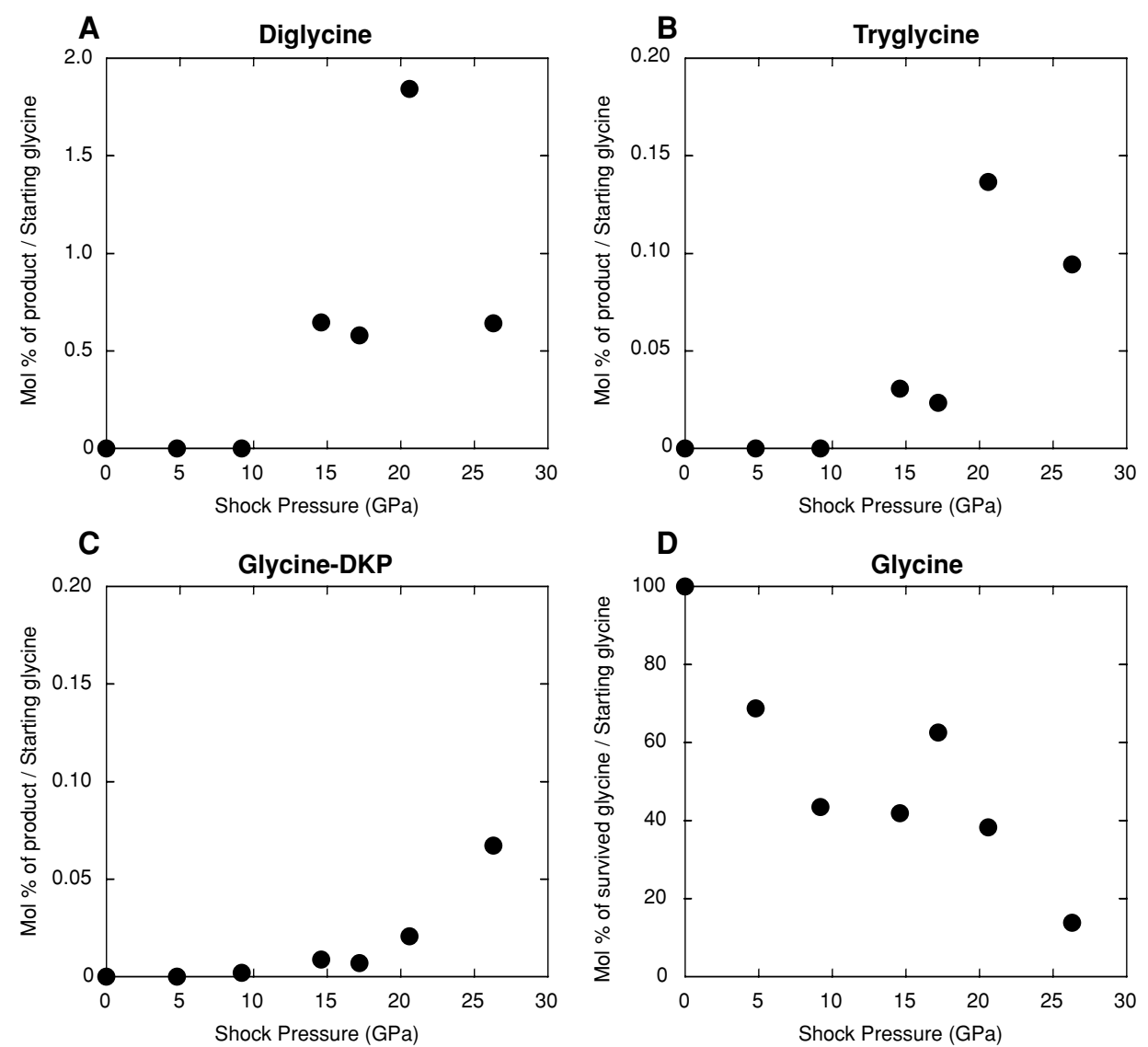

Fig. 3. The yields of peptides vs. shock pressure for diglycine (A), triglycine (B), glycine-diketopiperazine (C), and the survival ratio of glycine $(D)$. The yield of each peptide is shown in mol\% of product vs. the starting amino acid (glycine), and the survival ratio of glycine is shown in mol\% of the survived amino acid vs. the starting amino acid.

ing into other by-products. One candidate is the soot-like material that was observed in the shocked samples, although we did not analyze this material further.

\section{Discussion}

Peptide synthesis by impact shock

The yields of linear peptides were higher than that of cyclic peptide in the pressure range utilized in this study (Table 1). The formation of linear peptides may have been important in promoting chemical evolution, since the formation of cyclic peptides would have instead likely retarded further growth of peptide chains due to their lower reactivity (Kawamura et al., 2009). Where peptides are formed by intermolecular condensation, the formation of linear peptides is thermodynamically less favored compared to the formation of cyclic peptides (Streitwieser $e t$ al., 1998). Thus, heating solutions of amino acids leads to the synthesis of significant amounts of cyclic diketopiperazines (Harada and Fox, 1958). In contrast, shock reactions of amino acids disfavor intramolecular condensation and lead to the preferential synthesis of linear peptides, which combine to form more complex organic compounds. The reason for the differences recorded in the peptides resulting from heating and impact shock experiments may be due to the extremely short heating time or the high pressure of impact shock.

The condition of the starting material would influence the shock chemistry of amino acids. Our results of shock experiments performed on frozen amino acids at $77 \mathrm{~K}$ demonstrate the predominant synthesis of linear peptide forms, up to tripeptides, although these results are in contrast with the results of shock experiments on aqueous amino acids at room temperature, obtained by Blank et al. (2001). They noted that the yields of linear peptides (dipeptides) would be comparable in magnitude to those of cyclic peptides (diketopiperazines) by investigating at their respective peaks on the chromatograms of shocked samples. Tripeptides were not detected in their shocked samples, even though their starting amino acid concentrations were comparable to ours. The shock temperature at the peak-shocked state, the initial temperature, and the 
phase of water in the initial state are thought to be significant factors with respect to the differences observed between the results of the two experiments. Because the shock reaction is a thermal reaction (e.g., Greene and Toennies, 1964), the shock temperature should be the most important factor. However, the shock temperatures of two experiments do not differ significantly due to the similarity between the shock Hugoniots of water ice and liquid water (Stewart and Ahrens, 2005). Therefore, other reactions significantly influenced by the initial temperature and water phase may have caused the differences observed between the results of the two experiments. At the present stage, it is difficult to specify the reactions, but the special reaction at shock front may be one of the candidates. The reaction at the shock front is considered to be important in shock chemistry, in addition to the thermal reaction at the peak-shocked state (Greene and Toennies, 1964). The shock front represents a transitional region of the shocked material after the wave propagates. In this region, the particles are accelerated by the shock wave, causing the pressure and temperature of the material to increase dynamically. The particular effects caused by the non-equilibrium phenomena at the shock front are known to accelerate chemical reactions (e.g., Greene and Toennies, 1964; Genich et al., 1990; Kulikov, 1999; Velikodnyi and Kurochkin, 2002). The difference in the initial conditions (the phase of water and temperature) may have influenced the reaction at the shock front, which then could have caused the difference in the resulting peptides.

There is also a difference between the compositions of the starting materials used in our experiment and that by Blank et al. (2001). Our starting material contained forsterite and water ice as the matrix, while the starting material used by Blank et al. (2001) did not contain forsterite, and only made of liquid water. It is reported that the use of forsterite in pulverized basaltic lava does not show catalytic activity for the oligomerization of amino acids in heating experiments (Basiuk et al., 1998). If the oligomerization of amino acids by impact shock is mainly controlled by heating at the peak-shocked state, the use of forsterite does not affect the formation of linear peptides as a catalyst. However, as discussed in the former paragraph, chemical reactions are known to be promoted at the shock front (e.g., Greene and Toennies, 1964; Genich et al., 1990; Kulikov, 1999; Velikodnyi and Kurochkin, 2002). Thus, a control experiment (shock experiment at $77 \mathrm{~K}$, without forsterite) to examine the role of forsterite in the reaction at the shock front would be necessary in future research.

\section{Implications for cosmochemistry}

The present study demonstrated the potential of comet impacts to act as a driving force for peptide formation on the early Earth. This result is in contrast with the general view of comet impacts as destructive events. It is generally accepted that comet collisions with the Earth were more frequent in Earth's early history than today (e.g., Chyba and Sagan, 1992; Ryder, 2002; Morbidelli et al., 2012). Moreover, lunar cratering records suggest that the collisions of extraterrestrial bodies with the Earth were quite frequent in the period between $4.5 \times 10^{9}$ and $3.8 \times$ $10^{9}$ years ago, which is called the Late Heavy Bombardment (LHB) (e.g., Hartmann, 1966; Ryder, 2002). Although whether a catalytic spike existed in the record of impact frequency is an ongoing debate, recent research on the dynamical evolution of the Solar System, which is known as the Nice model, explains that the trigger of the LHB was the late migration of giant planets (Gomes et al., 2005; Morbidelli et al., 2005; Tsiganis et al., 2005). Several ideas have been proposed regarding the impactors of the LHB. Gomes et al. (2005) proposed that the main impactors were comets from the external icy disk and their calculated cometary mass accreted by the Earth is consistent with the upper limits of the cometary contribution to the current ocean mass, which is supposed from $\delta \mathrm{D}$ values. Meanwhile, Storm et al. (2005) and Bottke et al. (2012) proposed that the impactors originated mainly in the asteroid belt. The total mass accreted by the Earth during the LHB is calculated to be $8 \times 10^{22} \mathrm{~g}$ using the lunar cratering record (Levison et al., 2001). Although the proportion of comets in the LHB impactors is controversial, a significant amount of comets would have impacted the early Earth.

Considering the shock pressure of natural comet impacts, comets with the average impact velocity of the observed short-period comets $(24 \mathrm{~km} / \mathrm{s})$ (Hughes and Williams, 2000) generate pressures of more than $200 \mathrm{GPa}$ when impacting the Earth's ocean vertically. Such pressures are higher than the shock pressures of our experiments and are beyond the application of our results. However, there are many plausible scenarios that can reduce the shock pressure of comet impacts sufficiently. One scenario is the deceleration of comets by aerodynamic drag and airburst in the Earth's atmosphere. Deceleration by aerodynamic drag works more effectively on smaller objects. The numerical simulation by Bland and Artemieva (2006) showed that stony asteroids with diameters less than $1 \mathrm{~km}$ are decelerated, ablated, and easily fragmentized in the atmosphere. Further, impact velocity is significantly reduced by object fragmentation. This notion would be applicable to comets, as well. Another scenario is the reduction of shock pressure by oblique impacts. Fifty percent of extraterrestrial bodies impact the Earth with an impact angle of $30^{\circ}$ to $60^{\circ}$, resulting in shock pressure reductions of 58\% to $13 \%$ (Pierazzo and Melosh, 2000). Additionally, the shock pressure is distributed heterogeneously throughout an impacting ob- 
ject; the pressure at various points inside an impacting comet may be smaller than the pressure at the point of impact (Pierazzo and Melosh, 2000). Thus, taking into account all of these possibilities, comet impacts in the pressure range of our experiments are conceivable. The discussion presented above suggests that a significant number of comets impacted the early Earth in the pressure range of our experiments, and thus, a significant amount of comet-synthesized peptides were supplied to the early Earth.

The shock-synthesized peptides would have then spread over the early Earth, becoming important seeds for further chemical evolution. One suitable place for the further elongation of shock-synthesized peptides on the early Earth would have been submarine hydrothermal vents. The heat from the vent and the circulation of water from cold to hot regions would have supplied energy for further elongation of the peptide chains (e.g., Martin et al., 2008). It is noteworthy that the elongation of peptide chains by the addition of amino acids to dipeptides and tripeptides is thermodynamically a significantly easier process to carry out than the formation of dipeptides from two amino acids (Martin, 1998). Thus, the formation of an initial dipeptide, which is a type of nucleation event, is a critical component of abiotic peptide synthesis. Comet impacts might have been an important process in coming the first step of peptide synthesis on the early Earth.

Comet impacts are ubiquitous phenomena in the solar system: thus, peptide synthesis by comet impacts must have occurred on other extraterrestrial bodies, such as the icy satellites of Jupiter and Saturn (e.g., Europa and Enceladus). Because icy satellites were formed by the accumulation of comet-like materials (e.g., Canup and Ward, 2006), the satellites should contain appreciable amounts of peptides synthesized by the comet impacts. In particular, the intense bombardment of comets is considered to have occurred in the early Jovian system, as on the early Earth (Zahnle et al., 1998). Further, recent research has revealed that both Europa and Enceladus have subsurface oceans with several potential energy sources, such as tidal energy and radiogenic heating, which prevent complete freezing (e.g., Sohl et al., 2010). These extra energy sources make the two satellite environments suitable not only for the further elongation of peptide chains but also for the subsequent chemical evolution of peptides to more complex organic compounds. Cometary-driven peptides might have been an important source for chemical evolution on these icy satellites.

\section{CONCLUSIONS}

We conducted shock experiments on a mixture of glycine, water ice, and forsterite at cryogenic conditions (77 K) to simulate comet impacts. Our study demonstrated that impact shock synthesizes linear peptides up to trimer forms from frozen aqueous amino acids at $77 \mathrm{~K}$. Furthermore, linear peptides were preferentially synthesized relative to cyclic peptides. These results are in contrast with that of previous similar shock experiments performed at room temperature (Blank et al., 2001), indicating that the existence of cryogenic conditions is a key for the production of linear peptides. Our findings suggest that comet impacts can readily account for the oligomerization of amino acids, thereby forming the precursors of life on the early Earth. Moreover, because comet impacts are common phenomena in the solar system, such impacts most likely played an important role in organic chemical evolution on other extraterrestrial bodies, especially the icy satellites.

Acknowledgments-We thank M. Arakawa, S. Wallis, and S. Watanabe for fruitful discussions, E. Kawamura for supporting the calculations of the shock temperature and A. Nakamura for supporting the shock experiments carried out in cryogenic conditions. We also thank the members of the Instrument Development Center of School of Science, Nagoya University, for technical assistance. We are grateful for P. Claeys and anonymous reviewers for helpful comments. We acknowledge the Zonta club of Nagoya for funding. This work was supported by JSPS (Japan Society for the Promotion of Science) Research Fellowships for Young Scientists (228389) and a JSPS grant (21340164).

\section{REFERENCES}

Barbier, B., Visscher, J. and Schwartz, A. W. (1993) Polypeptide-assisted oligomerization of analogs in dilute aqueous solution. J. Mol. Evol. 37, 554-558.

Basiuk, V. A., Navarro-Gonzalez, R. and Basiuk, E. V. (1998) Behavior of amino acids when volatilized in the presence of silica gel and pulverized basaltic lava. Orig. Life Evol. Biosph. 28, 167-193.

Bernal, J. D. (1951) The Physical Basis of Life. Routledge, London.

Bernstein, M. P., Dworkin, J. P., Sandford, S. A., Cooper, G. W. and Allamandola, L. J. (2002) Racemic amino acids from the ultraviolet photolysis of interstellar ice analogues. $\mathrm{Na}$ ture 416, 401-403.

Bland, P. A. and Artemieva, N. A. (2006) The rate of small impacts on Earth. Meteorit. Planet. Sci. 42, 81-92.

Blank, J. G., Miller, G. H., Ahrens, M. J. and Winans, R. E. (2001) Experimental shock chemistry of aqueous amino acid solutions and the cometary delivery of prebiotic compounds. Orig. Life Evol. Biosph. 31, 15-51.

Botta, O., Martins, Z. and Ehrenfreund, P. (2007) Amino acids in Antarctic CM1 meteorites and their relationship to other carbonaceous chondrites. Meteorit. Planet. Sci. 42, 81-92.

Bottke, W. F., Vokrouhlicky, D., Minton, D., Nesvorny, D., Morbidelli, A., Brasser, R., Simonson, B. and Levison, H. (2012) An Archaean heavy bombardment from a destabilized extension of the asteroid belt. Nature 485, 78-81. 
Bujdák, J., Faybíková, K., Eder, A., Yongyai, Y. and Rode, B. M. (1995) Peptide chain elongation: A possible role of montmorillonite in prebiotic synthesis of protein precursors. Orig. Life Evol. Biosph. 25, 431-441.

Canup, R. M. and Ward, W. R. (2006) A common mass scaling for satellite systems of gaseous planets. Nature 441, 834839.

Charnley, S. B., Rodgers, S. D., Kuan, Y. J. and Huang, H. C. (2002) Adv. Space Res. 30, 1419-1431.

Chizhov, V. E. (1993) Thermodynamic properties and thermal equations of the state of high-pressure ice phases. J. Appl. Mech, Tech. Phys. 34, 253-262.

Chyba, C. F. and Sagan, C. (1992) Endogenous production, exogenous delivery and impact-shock synthesis of organic molecules; an inventory for the origin of life. Nature $\mathbf{3 5 5}$, $125-132$.

Corliss, J. B., Baross, J. A. and Hoffmann, S. E. (1981) An hypothesis concerning the relationship between submarine hot springs and the origin of life on Earth. Oceanologica Acta (Suppl.) 4, 59-69.

Cronin, J. R. and Pizzarello, S. (1983) Amino acids in meteorites. Adv. Space Res. 3, 5-18.

Deming, T. J. (2006) Polypeptide and polypeptide hybrid copolymer synthesis via NCA polymerization. Adv. Polym. Sci. 202, 1-18.

Duffy, T. S. and Ahrens, T. J. (1997) Dynamic compression of an Fe-Cr-Ni alloy to $80 \mathrm{GPa}$.J. Appl. Phys. 82, 4259-4269.

Ehrenfreund, P. and Charnley, S. B. (2000) Organic molecules in the interstellar medium. Comets, and meteorites: A voyage from dark clouds to the early Earth. Annu. Rev. Astron. Astrophys. 38, 427-483.

Ehrenfreund, P., Glavin, D. P., Botta, O., Cooper, G. and Bada, J. (2001) Extraterrestrial amino acids in Orgueil and Ivuna: Tracing the parent body of CI type carbonaceous chondrites. Proc. Natl. Acad. Sci. U.S.A. 98, 2138-2141.

Elsila, J. E., Gravin, D. P. and Dworkin, J. P. (2009) Cometary glycine detected in samples returned by Stardust. Meteorit. Planet. Sci. 44, 1323-1330.

Flores, J. J. and Leckie, J. O. (1973) Peptide formation mediated by cyanate. Nature 12, 435-437.

Fox, S. W. (1956) Evolution of protein molecules and thermal synthesis of biochemical substances. Am. Sci. 44, 347-359.

Fox, S. W. and Harada, K. (1958) Thermal copolymerization of amino acids to a product resembling protein. Science $\mathbf{1 2 8}$, 1214.

Furukawa, Y., Sekine, T., Oba, M., Kakegawa, T. and Nakazawa, H. (2009) Biomolecule formation by oceanic impacts on early Earth. Nature Geosci. 2, 62-66.

Genich, A. P., Kulikov, S. V., Manelis, G. B., Myagkov, Yu. P. and Chereshnev, S. L. (1990) Translational nonequilibrium effects in shock waves in gases. Fluid Dyn. 25, 443-448.

Goldman, N., Reed, E. J., Fried, L. E., Kuo, I. F. W. and Maiti, A. (2010) Synthesis of glycine-containing complexes in impacts of comets on the early Earth. Nature Chem. 2, 949954.

Gomes, R., Levison, H. F., Tsiganis, K. and Morbidelli, A. (2005) Origin of the cataclysmic Late Heavy Bombardment period of the terrestrial planets. Nature 435, 466-469.

Greenberg, J. M. and Li, A. (1999) Morphological structure and chemical composition of cometary nuclei and dust. Space Sci. Rev. 90, 149-161.

Greene, E. F. and Toennies, J. P. (1964) Chemical Reactions on Shock Waves. Edward Arnold, Ltd., London.

Harada, K. and Fox, S. (1958) The thermal condensation of glutamic acid and glycine to linear peptides. J. Am. Chem. Soc. 80, 2694-2697.

Hartmann, W. K. (1966) Early Lunar cratering. Icarus 5, 406418.

Holton, P. D., Bennett, C. J., Osamura, Y., Mason, N. J. and Kaiser, R. I. (2005) A combined experimental and theoretical study on the formation of the amino acid glycine $\left(\mathrm{NH}_{2} \mathrm{CH}_{2} \mathrm{COOH}\right)$ and its isomer $\left(\mathrm{CH}_{3} \mathrm{NHCOOH}\right)$ in extraterrestrial ices. Astrophys. J. 626, 940-952.

Huber, C. and Wächtershäuser, G. (1998) Peptides by activation of amino acids with $\mathrm{CO}$ on $(\mathrm{Ni}, \mathrm{Fe}) \mathrm{S}$ surfaces: implications for the origin of life. Science 281, 670-672.

Huber, C., Eisenreich, W., Hecht, S. and Wächtershäuser, G. (2003) A possible primordial peptide cycle. Science 301, 938-940.

Hughes, D. W. and Williams, I. P. (2000) The velocity distributions of periodic comets and stream meteoroids. Mon. Not. R. Astron. Soc. 315, 629-634.

Imai, E., Honda, H., Hatori, K., Brack, A. and Matsumoto, K. (1999) Elongation of oligopeptides in a simulated submarine hydrothermal system. Science 283, 831-833.

Kawamura, K., Takeya, H. and Kushibe, T. (2009) Effect of condensation agents and minerals for oligopeptide formation under mild and hydrothermal conditions in related to chemical evolution of proteins. Adv. Space Res. 44, 267275.

Kobayashi, K., Takano, Y., Masuda, H., Tonishi, H., Kaneko, T., Hashimoto, H. and Saito, T. (2004) Possible cometary organic compounds as sources of planetary biospheres. $A d v$. Space Res. 33, 1277-1281.

Kochavi, E., Bar-Num, A. and Fleminger, G. (1997) Substratedirected formation of small biocatalysts under prebiotic conditions. J. Mol. Evol. 45, 342-351.

Kraus, P. G., Stewart, S. T., Seifter, A. and Obst, A. W. (2010) Shock and post-shock temperatures in an ice-quartz mixture: implications for melting during planetary impacts events. Earth Planet. Sci. Lett. 289, 162-170.

Kulikov, S. V. (1999) Acceleration of chemical reactions within a shock wave front. Shock Waves 9, 413-417.

Kvenvolden, K., Lawless, J., Pering, K. M., Peterson, E., Flores, J., Ponnamperuma, C., Kaplan, I. R. and Moore, C. (1970) Evidence for extraterrestrial amino-acids and hydrocarbons in the Murchison meteorite. Nature 228, 923-926.

Lambert, J. F. (2008) Adsorption and polymerization of amino acids on mineral surfaces: a review. Orig. Life Evol. Biosph. 38, 211-242.

Larouche, S., Marsh, E. T. and Mikkola, D. E. (1981) Strengthening effects of deformation twins and dislocations introduced by short duration shock pulses in $\mathrm{Cu}-8.7 \mathrm{Ge}$. Metall. Trans. A 12A, 1777-1785.

Leadbetter, A. J. (1965) The thermodynamic and vibrational properties of $\mathrm{H}_{2} \mathrm{O}$ Ice and $\mathrm{D}_{2} \mathrm{O}$ ice. Proc. R. Soc. A 287, 403-425.

Lemke, K. H., Rosenbauer, R. J. and Bird, D. K. (2009) Pep- 
tide synthesis in early Earth hydrothermal systems. Astrobiology 9, 141-146.

Leuchs, H. (1906) Under die Glycin-carbonsäure. Berichte der deutschen chemischen Gesellschaft 39, 857-861.

Levison, H. F., Dones, L., Chapman, C. R. and Stern, A. (2001) Could Lunar "Late Heavy Bombardment" have been triggered by the formation of Uranus and Neptune? Icarus $\mathbf{1 5 1}$, 286-306.

Marsh, S. P. (1980) LASL Shock Hugoniot Data. University of California Press, Berkeley.

Martin, R. B. (1998) Free energies and equilibria of peptide bond hydrolysis and formation. Biopolymers 45, 351-353.

Martin, W., Baross, J., Kelley, D. and Russell, M. J. (2008) Hydrothermal vents and the origin of life. Nature Rev. Microbiol. 6, 805-814.

Martins, Z., Alexander, C. M. O’D., Orzechowska, G. E., Fogel, M. L. and Ehrenfreund, P. (2007) Indigenous amino acids in primitive CR meteorites. Meteorit. Planet. Sci. 42, 21252136.

Mashimo, T., Nishii, K., Soma, T. and Sawaoka, A. (1980) Some physical properties of amorphous $\mathrm{SiO}_{2}$ synthesized by shock compression of $\alpha$ quartz. Phys. Chem. Minerals 5, 367-377.

McPherson, D. W., Rahman, K., Martinez, I. and Shevlin, P. B. (1987) The formation of amino acid precursors in the reaction of atomic carbon with water and ammonia at $77 \mathrm{~K}$. Orig. Life 17, 275-282.

Meyers, M. A. (1994) Dynamic Behavior of Materials. John Wiley, New York.

Mimura, K. and Toyama, S. (2005) Behavior of polycyclic aromatic hydrocarbons at impact shock: Its implication for survival of organic materials delivered to the early Earth. Geochim. Cosmochim. Acta 69, 201-209.

Mimura, K., Arao, T., Sugiura, M. and Sugisaki, R. (2003) Shock-induced carbonization of phenanthrene at pressures of 7.9-32 GPa. Carbon 41, 2547-2553.

Morbidelli, A., Levison, H. F., Tsiganis, K. and Gomes, R. (2005) Chaotic capture of Jupiter's Trojan asteroids in the early Solar System. Nature 435, 462-465.

Morbidelli, A., Marchi, S., Bottke, W. F. and Kring, D. A. (2012) A sawtooth-like timeline for the first billion years of lunar bombardment. Earth Planet. Sci. Lett. 355, 144-151.

Muñoz Caro, G. M., Meierhenrich, U. J., Schutte, W. A., Barbier, B., Arcones Segovia, A., Rosenbauer, H., Thiemann, W. H.P., Brack, A. and Greenberg, J. M. (2002) Amino acids from ultraviolet irradiation of interstellar ice analogues. Nature 416, 403-406.

Orava, R. N. and Wittman, R. H. (1975) Techniques for the control and application of explosive shock waves. Proc. 5th International Conference on High Energy Rate Fabrication, pp. 1.1.1. University of Denver Research Institute, Denver, Colorado.

Oró, J. and Guidry, C. L. (1960) A novel synthesis of polypeptides. Nature 186, 156-157.

Oró, J., Gibert, J., Lichtenstein, H., Wikstrom, S. and Flory, D. A. (1971) Amino acids, aliphatic hydrocarbons in the Murchison meteorite. Nature 230, 105-106.

Pierazzo, E. and Melosh, H. J. (2000) Hydrocode modeling of oblique impact: the fate of the projectile. Meteoritics Planet. Sci. 35, 117-130.
Pizzarello, S. and Weber, A. L. (2010) Stereoselective syntheses of pentose sugars under realistic prebiotic conditions. Orig. Life Evol. Biosph. 40, 3-10.

Ryder, G. (2002) Mass flux in the ancient Earth-Moon system and benign implications for the origin of life on Earth. $J$. Geophys. Res. 107, 1-13.

Schreiner, E., Nair, N. N. and Marx, D. (2009) Peptide synthesis in aqueous environment: The role of extreme conditions on peptide bond formation and peptide hydrolysis. J. Am. Chem. Soc. 131, 13668-13675.

Shimizu, M. (1995) Specific aminoacylation of C4N hairpin RNAs with the cognate aminoacyl-adenylates in the presence of a dipeptide: Origin of the genetic code. J. Biochem. 117, 23-26.

Shimoyama, A. and Ogasawara, R. (2002) Dipeptides and diketopiperazines in the Yamato-791198 and Murchison carbonaceous chondrites. Orig. Life Evol. Biosph. 32, 165179.

Shock, E. L. (1992) Hydrothermal organic synthesis experiments. Orig. Life Evol. Biosph. 22, 135-146.

Simakov, M. B., Kuzicheva, E. A., Mal'ko, I. L. and Dodonova, N. Ya. (1996) Abiogenic synthesis of oligopeptides in solid state under action of vacuum ultraviolet light (100-200 nm). Adv. Space Res. 18, 61-64.

Simakov, M. B., Kuzicheva, E. A., Dodonova, N. Ya. and Antropov, A. E. (1997) Formation of oligopeptides on the surface of small bodies in solar system by cosmic raddiacion. Adv. Space Res. 19, 1063-1066.

Sohl, F., Choukroun, M., Kargel, J., Kimura, J., Pappalardo, R., Vance, S. and Zolotov, M. (2010) Subsurface water oceans on icy satellites: chemical composition and exchange processes. Space Sci. Rev. 153, 485-510.

Stewart, S. T. and Ahrens, T. J. (2005) Shock properties of $\mathrm{H}_{2} \mathrm{O}$ ice. J. Geophys. Res. 110, E03005.

Storm, R. G., Malhotra, R., Ito, T., Yoshida, F. and Kring, D. A. (2005) The origin of planetary impactors in the inner solar system. Science 309, 1847-1850.

Streitwieser, A., Heathcock, C. H. and Kosower, E. M. (1998) Amino acids, peptides, and proteins. Introduction to Organic Chemistry. 4th ed., 949-1003, Prentice Hall, Inc., U.S.A.

Sugisaki, R., Mimura, K. and Kato, M. (1994) Shock synthesis of light hydrocarbon gases from $\mathrm{H}_{2}$ and $\mathrm{CO}$ : Its role in astrophysical processes. Geophys. Res. Lett. 21, 1031-1034.

Tanaka, M., Kaneko, F., Kotetsu, T., Nakagawa, K. and Yamada, T. (2008) Fragmentation and dimerization of aliphatic amino acid film induced by vacuum ultraviolet irradiation. Radiat. Phys. Chem. 77, 1164-1168.

Tomeoka, K., Tomioka, N. and Ohnishi, I. (2008) Silicate minerals and $\mathrm{Si}-\mathrm{O}$ glass in comet Wild 2 samples: Transmission electron microscopy. Meteorit. Planet. Sci. 43, 273284.

Tsiganis, K., Gomes, R., Morbidelli, A. and Levison, H. F. (2005) Origin of the orbital architecture of the giant planets of the solar system. Nature 435, 459-461.

van der Gulik, P., Massar, S., Gilis, D., Buhrman, H. and Rooman, M. (2009) The first peptides: The evolutionary transition between prebiotic amino acids. J. Theoret. Biol. 261, 531-539. 
Velikodnyi, V. Yu. and Kurochkin, V. I. (2002) The rate of nonequilibrium physicochemical processes in the shock wave front in a dense gas mixture. High Temperature 40, 670-676.

Wang, W., Yuan, H., Wang, X. and Yu, Z. (2007) keV ion irradiation assisted prebiotic synthesis of oligopeptide in the solar system. Adv. Space Res. 40, 1641-1645.

Weber, A. and Pizzarello, S. (2006) The peptide-catalyzed ster- eospecific synthesis of tetroses: A possible model for prebiotic molecular evolution. Proc. Natl. Acad.Sci. U.S.A. 103, 12713-12717.

Yanagawa, H., Nishizawa, M. and Kojima, K. (1984) A possible prebiotic peptide formation from glycinamide and related compounds. Orig. Life 14, 267-272.

Zahnle, K., Dones, L. and Levison, H. F. (1998) Cratering rates on the Galilean satellites. Icarus 136, 202-222. 